\title{
Temperature and composition dependence of crystal structures and magnetic and electronic properties of the double perovskites $\mathrm{La}_{2-x} \mathrm{Sr}_{x} \mathrm{CoIrO}_{6}(0 \leq x \leq 2)$
}

\author{
N. Narayanan, ${ }^{1,2, *}$ D. Mikhailova, ${ }^{1,2}$ A. Senyshyn, ${ }^{1}$ D. M. Trots, ${ }^{3}$ R. Laskowski, ${ }^{4}$ P. Blaha,,${ }^{4}$ K. Schwarz, ${ }^{4}$ H. Fuess, ${ }^{1}$ and \\ H. Ehrenberg 1,2 \\ ${ }^{1}$ Institute for Materials Science, Darmstadt University of Technology, Petersenstr. 23, D-64287, Darmstadt, Germany \\ ${ }^{2}$ Institute for Complex Materials, IFW Dresden, Helmholtzstr. 20, D-01069 Dresden, Germany \\ ${ }^{3}$ Bayerisches Geoinstitut, Universität Bayreuth, D-95440 Bayreuth, Germany \\ ${ }^{4}$ Institute for Materials Chemistry, Vienna University of Technology, Getreidemarkt 9/165-TC, A-1060 Vienna, Austria
}

(Received 12 February 2010; revised manuscript received 10 June 2010; published 6 July 2010)

\begin{abstract}
$\mathrm{X}$-ray, synchrotron, and neutron powder diffraction techniques were combined to investigate the evolution of crystal structure and physical properties of $\mathrm{La}_{2-x} \mathrm{Sr}_{x} \mathrm{CoIrO}_{6}$ with temperature and composition $x$. The following sequence of first- and second-order phase transitions is observed in this system, induced by increasing $\mathrm{Sr}$ content and temperature: $P 2_{1} / n \leftrightarrow P 2_{1} / n+I 2 / m \leftrightarrow I 2 / m \leftrightarrow I 4 / m \leftrightarrow F m \overline{3} m$. The low-temperature magnetic structures are characterized by the propagation vector $k=(0,0,0)$ for $x=0, k=(1 / 2,0,1 / 2)$ for $x=1$, and $k$ $=(0,1 / 2,1 / 2)$ or $k=(1 / 2,0,1 / 2)$ for 1.5 and 2 . Different noncollinear magnetic structures are concluded from the combination of magnetization measurements and neutron powder diffraction. Resistivity measurements reveal that the whole series behaves like nonmetals with electronic transport described by a combination of thermal activation and variable range hopping. Band gaps determined by electronic structure calculations agree very well with the experimental data for $x=0$ and 1 , and the calculated occupation of the $d$ bands of Co and Ir are in good agreement with a transition IS/ $\mathrm{HS}_{-} \mathrm{Co}^{2+} / \mathrm{LS}-\mathrm{Ir}^{4+} \rightarrow \mathrm{HS}-\mathrm{Co}^{3+} / \mathrm{LS}^{2} \mathrm{Ir}^{5+}$ with increasing $\mathrm{Sr}$ content.
\end{abstract}

DOI: $10.1103 /$ PhysRevB.82.024403

PACS number(s): 75.25.-j, 71.20.-b, 61.50.Ks

\section{INTRODUCTION}

In recent years double perovskites $A_{2} B B^{\prime} \mathrm{O}_{6}$ with $3 d$ and $5 d$ transition metals at $B$ and $B^{\prime}$ sites, respectively, have been extensively studied due to their interesting physical properties such as colossal magnetoresistance, high Curie temperatures, or metal-insulator transition. ${ }^{1-3}$ Most of the research was focused on $B^{\prime}=\mathrm{W}-$, Re-, and Os-based double perovskites with ferromagnetic ordering temperatures $T_{\mathrm{C}}$ above room temperature. Less attention was diverted toward the Ir-based ones, although the ability of Ir to exist in different oxidation states and the spatially more extended $5 d$ orbitals should result in a rich variety of physical properties and elucidate the structure-property relationships in this interesting class of compounds. Ir-based double perovskites $\mathrm{La}_{2} B \mathrm{IrO}_{6}$, $B=\mathrm{Mg}, \mathrm{Mn}, \mathrm{Co}, \mathrm{Ni}$, and $\mathrm{Cu}$, were first synthesized in 1965. ${ }^{4,5}$ Much later crystal structures and magnetic properties were determined for $B=\mathrm{Mn}, \mathrm{Co}, \mathrm{Ni}$, and $\mathrm{Zn}$ and bandstructure calculations [only local-density approximation (LDA)] were performed for $B=\mathrm{Mn}, \mathrm{Co}$, and $\mathrm{Fe} .{ }^{6-8}$ At room temperature the double perovskites with $B=\mathrm{Co}, \mathrm{Ni}$, and $\mathrm{Zn}$ adopt a monoclinic $P 2_{1} / n$ superstructure while the underlying space group of $\mathrm{La}_{2} \mathrm{MnIrO}_{6}$ was not determined. A high degree of cation disorder, i.e., an occupation of the $B$ site with $B^{\prime}$ and vice versa, was also reported for the compounds $\mathrm{La}_{2} B \mathrm{IrO}_{6}$ with $B=\mathrm{Mn}$ and $\mathrm{Fe}$ due to the small differences in charge and ionic radii between the $B$ and $B^{\prime}=\mathrm{Ir}$ ions. ${ }^{7}$ Ferromagnetic behavior is reported for $\mathrm{La}_{2} \mathrm{MnIrO}_{6}$, based on magnetization measurements and confirmed by bandstructure calculations. A more complicated behavior was found for $B=\mathrm{Co}, \mathrm{Ni}$, and $\mathrm{Fe}$ : The magnetic ground states of these compounds exhibit both ferromagnetic and antiferromagnetic components, so that noncollinear magnetism (NCM) was concluded, again supported by calculations, which gave larger calculated magnetic moments for $B=\mathrm{Co}$ and $\mathrm{Fe}$ in a collinear framework than the observed ones. ${ }^{7-9} \mathrm{~A}$ well-established method to affect the physical properties of double perovskites is the partial substitution of cations. ${ }^{10,11}$ In this work only on $A$-site substitution of $\mathrm{La}$ by $\mathrm{Sr}$ is considered, which has mainly two effects on the resulting structures and properties. On one hand the La:Sr ratio determines the averaged ionic size on the $A$ site and hereby the symmetry of the underlying crystal structure. On the other hand the replacement of trivalent $\mathrm{La}$ by bivalent $\mathrm{Sr}$ increases the formal oxidation states on $B$ and $B^{\prime}$ sites. This means a lower number of $d$ electrons, and therefore less repulsion between these $d$ electrons, which could result in an insulator to metal transition. The $\mathrm{La}_{2-x} \mathrm{Sr}_{x} \mathrm{CoIrO}_{6}$ system offers therefore two degrees of freedom, which are correlated with each other. In addition, however, the cation-site disorder $\mathrm{Co} \leftrightarrow \operatorname{Ir}$ on $B$ and $B^{\prime}$ sites $(y)$ and a possible oxygen deficiency $\delta$ will also affect the physical properties and have to be considered carefully as two additional relevant parameters in the system $\mathrm{La}_{2-x} \mathrm{Sr}_{x}\left(\mathrm{Co}_{1-y} \mathrm{Ir}_{y}\right)\left(\operatorname{Ir}_{1-y} \mathrm{Co}_{y}\right) \mathrm{O}_{6-\delta}$.

\section{EXPERIMENTAL}

Polycrystalline samples of $\mathrm{La}_{2-x} \mathrm{Sr}_{x} \mathrm{CoIrO}_{6}$ were synthesized by solid-state reaction for five different compositions $x=0,0.5,1,1.5$, and 2. Stoichiometric amounts of reactants $\mathrm{La}_{2} \mathrm{O}_{3}, \mathrm{SrCO}_{3}, \mathrm{CoO}$, and $\mathrm{IrO}_{2}$ were ground together in an agate mortar, pressed into pellets, placed into corundum crucibles, heated to $1200{ }^{\circ} \mathrm{C}$ in air within a muffle furnace, hold at this temperature for $24 \mathrm{~h}$ and slowly cooled down to room temperature. Under these conditions of synthesis only negligible oxygen deficiency $\delta \approx 0$ is expected. Phase purity and crystal structures were determined at room temperature by 
TABLE I. Nonoverlapping atomic sphere radii (in a.u.), mesh of the special $k$ points in the IBZ, and the number of independent atoms corresponding to composition $x$ and the different magnetic structures.

\begin{tabular}{|c|c|c|c|c|c|c|c|c|}
\hline$x$ & $\mathrm{La}$ & $\mathrm{Sr}$ & $\mathrm{Co}$ & Ir & $\mathrm{O}$ & Special $k$ mesh & $\begin{array}{c}\text { No. of } \\
\text { independent atoms }\end{array}$ & Magnetic structure \\
\hline \multirow[t]{3}{*}{0} & 2.38 & & 2.04 & 1.98 & 1.76 & $6 \times 6 \times 4$ & 6 & FM \\
\hline & & & & & & $6 \times 6 \times 4$ & 12 & $k=(0,0,0), \mathrm{AFM}$ \\
\hline & & & & & & $4 \times 2 \times 1$ & 80 & $k=(0,1 / 2,1 / 2), \mathrm{AFM}$ \\
\hline $0.5^{\mathrm{a}}$ & 2.40 & 2.26 & 2.06 & 1.97 & 1.75 & $5 \times 5 \times 3$ & 20 & FM \\
\hline \multirow[t]{2}{*}{1.0} & 2.40 & 2.27 & 2.02 & 1.96 & 1.73 & $2 \times 4 \times 1$ & 80 & $k=(1 / 2,0,1 / 2), \mathrm{AFM}$ \\
\hline & & & & & & $4 \times 2 \times 1$ & 80 & $k=(0,1 / 2,1 / 2), \mathrm{AFM}$ \\
\hline $1.5\left(P 2_{1} / n\right)^{\mathrm{a}}$ & 2.37 & 2.24 & 2.00 & 1.91 & 1.70 & $5 \times 5 \times 3$ & 20 & FM \\
\hline $1.5(I 2 / m)^{\mathrm{a}}$ & 2.50 & 2.32 & 1.89 & 1.91 & 1.68 & $5 \times 5 \times 3$ & 16 & FM \\
\hline $2.0\left(P 2_{1} / n\right)$ & & 2.38 & 1.91 & 1.92 & 1.69 & $4 \times 2 \times 1$ & 80 & $k=(0,1 / 2,1 / 2), \mathrm{AFM}$ \\
\hline $2.0(I 2 / m)$ & & 2.38 & 1.91 & 1.94 & 1.70 & $4 \times 2 \times 1$ & 80 & $k=(0,1 / 2,1 / 2), \mathrm{AFM}$ \\
\hline
\end{tabular}

${ }^{\mathrm{a} O n l y}$ for the calculation of Bader charges.

$\mathrm{X}$-ray powder diffraction in flat-sample transmission mode, using a STOE STADI $\mathrm{P}$ diffractometer, operated with Mo- $K \alpha_{1}$ radiation and equipped with a linear position sensitive detector. The temperature dependence of the crystal structures was investigated by synchrotron powder diffraction at the beamline B2 (Ref. 12) of HASYLAB/DESY (Hamburg, Germany) using the onsite readable image-plate detector OBI, ${ }^{13}$ a STOE furnace equipped with a EUROTHERM temperature controller for high-temperature measurements $[\lambda=0.49327(1) \AA]$ and a special cryostat $^{14}$ for low-temperature measurements $[\lambda=0.50205(1) \AA]$. Neutron powder diffraction [NPD, $\lambda=1.548(1) \AA]$ was performed at the SPODI instrument of FRM II (Garching n. Munich, Germany) at selected temperatures to refine oxygen positions and site-specific occupations very reliably and to determine the magnetic structures below the magnetic-ordering temperatures. Rietveld refinements of structural parameters were performed, based on synchrotron- and neutron-diffraction data simultaneously, using the program FULLPROF. ${ }^{15}$ The propagation vectors of the magnetic structures were determined with the program SUPERCELL. Initial structure models were taken from Woodward. ${ }^{16}$ The temperature dependence of magnetization was measured both in zero-field-cooled (ZFC) mode and in field-cooled (FC) mode over the temperature range from 5 to $350 \mathrm{~K}$ and the field dependence of magnetization up to $7 \mathrm{~T}$ using a superconducting quantum interference device [SQUID magnetometer (MPMS) magnetic properties measurement system from Quantum Design]. The resistivity measurements were performed using a physical property measurement system from Quantum Design in the van der Pauw four point setup.

\section{ELECTRONIC STRUCTURE CALCULATIONS}

First-principles density-functional theory (DFT) spinpolarized electronic structure calculations were performed for $x=0,1$, and 2 (calculations were performed for $x=0.5$ and 1.5 in the ferromagnetic (FM) configuration only to obtain the Bader charges) by the full-potential linearized augmented plane wave plus local orbital method as implemented in the WIEN2K code. ${ }^{17}$ The following nonoverlapping atomic spheres with radii $R_{\mathrm{MT}}$ (in a.u.) were used (Table I).

The value of $R_{\mathrm{MT}} K_{\max }$, where $K_{\max }$ corresponds to the largest plane-wave vector, was set to 6.5 and spherical harmonics up to the tenth order were included in the expansion of the radial part of the wave function. The mesh of special $k$ points in the irreducible Brillouin zone (IBZ) are selected as shown in Table I. The numbers of independent atoms in the unit cell are also given in Table I. For the exchangecorrelation energy functional the Perdew-Burke-Ernzerhof

TABLE II. Space groups and structural parameters of $\mathrm{La}_{2-x} \mathrm{Sr}_{x} \mathrm{CoIrO}_{6}$ at $3 \mathrm{~K}$.

\begin{tabular}{lccccc}
\hline \hline \multicolumn{1}{c}{} & Space group & $\begin{array}{c}a \\
(\AA)\end{array}$ & $\begin{array}{c}b \\
(\AA)\end{array}$ & $\begin{array}{c}c \\
(\AA)\end{array}$ & $\begin{array}{c}\beta \\
(\mathrm{deg})\end{array}$ \\
\hline 0 & $P 2_{1} / n$ & $5.5684(1)$ & $5.6604(1)$ & $7.8956(2)$ & $89.96(1)$ \\
$0.5^{\mathrm{a}}$ & $P 2_{1} / n$ & $5.5979(1)$ & $5.6251(1)$ & $7.9112(1)$ & $90.004(5)$ \\
1.0 & $P 2_{1} / n$ & $5.6121(9)$ & $5.6015(6)$ & $7.9182(4)$ & $89.96(1)$ \\
$1.5^{\mathrm{a}}$ & $P 2_{1} / n$ & $5.5933(2)$ & $5.5549(3)$ & $7.9117(3)$ & $89.98(1)$ \\
$1.5^{\mathrm{a}}$ & $I 2 / m$ & $5.5564(3)$ & $5.5456(2)$ & $7.8022(3)$ & $90.24(1)$ \\
2.0 & $P 2_{1} / n$ & $5.5611(3)$ & $5.5351(3)$ & $7.7920(2)$ & $89.70(1)$ \\
2.0 & $I 2 / m$ & $5.5940(1)$ & $5.5560(1)$ & $7.7208(1)$ & $89.93(1)$ \\
\hline \hline
\end{tabular}

${ }^{\mathrm{a} O n l y}$ for the calculation of Bader charges. 
TABLE III. Space groups and structural parameters of $\mathrm{La}_{2-x} \mathrm{Sr}_{x} \mathrm{CoIrO}_{6}$ at room temperature.

\begin{tabular}{lcccccc}
\hline \hline & & & $a$ & $b$ & $c$ & $\beta$ \\
$x$ & Space group & $f$ & $(\AA)$ & $\begin{array}{c}b \\
(\AA)\end{array}$ & $(\AA)$ & $(\mathrm{deg})$ \\
\hline 0 & $P 2_{1} / n$ & 0.8682 & $5.5819(1)$ & $5.6576(1)$ & $7.9078(2)$ & $89.98(1)$ \\
0.5 & $P 2_{1} / n$ & 0.8868 & $5.6010(1)$ & $5.6151(1)$ & $7.9151(2)$ & $89.982(5)$ \\
1.0 & $P 2_{1} / n$ & 0.9058 & $5.5988(1)$ & $5.5750(1)$ & $7.9029(2)$ & $89.989(6)$ \\
1.5 & $P 2_{1} / n$ & 0.9253 & $5.5960(2)$ & $5.5613(4)$ & $7.9135(3)$ & $89.98(1)$ \\
& $I 2 / m$ & & $5.5633(3)$ & $5.5329(2)$ & $7.8479(5)$ & $89.94(1)$ \\
2.0 & $I 2 / m$ & 0.9452 & $5.5285(2)$ & $5.5508(2)$ & $7.8427(3)$ & $90.297(3)$ \\
\hline \hline
\end{tabular}

version of the generalized gradient approximation (GGA) was used. ${ }^{18}$ The spin-orbit-coupling [SOC (quantization axis: $z)]$ was included in a second variational step with scalar relativistic orbitals as basis. ${ }^{19}$ The structural parameters were taken from NPD at $3 \mathrm{~K}$ and are shown in Table II. The convergence is reached as the difference in the charge fluctuation becomes less than 0.0001 of an electron. Additionally a relaxation of the internal atomic positions is carried out for $x=0$ in order to compare the total energies of the FM $(4 \times 4 \times 2), k=(0,0,0)$ antiferromagnetic $(\mathrm{AFM})(4 \times 4 \times 2)$, and the $k=(0,1 / 2,1 / 2)$ AFM $(4 \times 2 \times 1)$ structures. From the converged charge densities Bader charges are also calculated for all five compositions using the Bader's AiM concept (using WIEN2K). ${ }^{20}$ For this calculation additionally to the charge densities of the experimentally found AFM structures of $x=0,1$, and 2, charge densities of the FM configuration of $x=0.5$ and 1.5 are used. However it should be mentioned, that this approach does not always give a quantitative assessment of the valence state but it is quite useful for investigating trends within a certain system. ${ }^{21}$ The trend of the Bader charges is discussed in Sec. IV A together with the bond valence sums (BVSs).

\section{RESULTS AND DISCUSSION}

\section{A. Room-temperature crystal structures}

All members of the series crystallize with a monoclinic symmetry but in two different space groups. Compounds with the composition $x=0,0.5$, and 1 crystallize in the space group $P 2{ }_{1} / n$. This agrees with the crystal structure reported earlier for $\mathrm{La}_{2} \mathrm{CoIrO}_{6}$ with $x=0 .{ }^{7} \mathrm{Sr}_{2} \mathrm{CoIrO}_{6}$ with $x=2$ crystallizes in the space group $I 2 / m$. For $x=1.5$ two monoclinic modifications with space groups $P 2_{1} / n$ and $I 2 / m$ coexist at
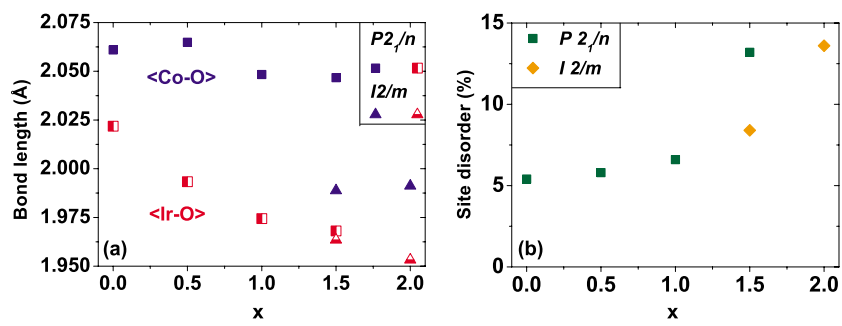

FIG. 1. (Color online) (a) Composition dependence of Co-O and Ir-O bond lengths and (b) composition dependence of the site disorder. room temperature. Depending on composition the following phase transitions occur at room temperature with increasing Sr content: $P 2_{1} / n \leftrightarrow P 2_{1} / n+I 2 / m \leftrightarrow I 2 / m$. The corresponding crystal structures are superstructures of the perovskite type. The deviation from the ideal cubic perovskite structure is the distribution of Co and Ir on two distinct crystallographic sites ("double perovskite") and rotations of the corner-sharing $\mathrm{CoO}_{6}$ and $\mathrm{IrO}_{6}$ octahedra, which break the cubic symmetry. (For a comparison of the crystal structures of the two monoclinic forms with space groups $P 2_{1} / n$ and $I 2 / m$ see Fig. 5.) Both structures differ with respect to the specific octahedra tilting system, described by Glazer's notation (Ref. 16) and belong to the twelve possible space groups derived group theoretically by Howard et al., ${ }^{10,22}$ by means of considering the deviation only due to the tilting of the octahedra. An empirical criterion for the prediction of the specifically distorted perovskite-type structure is based on the ionic radii according to the Goldschmidt tolerance factor $f .{ }^{23}$ For an ideal cubic perovskite-type structure $f$ should be very close to 1 , monoclinic crystal structures are expected for $f<0.97$ (Ref. 24). For all the compositions in this system $f$ is clearly less than 0.97 and thus fulfils the empirical rule. The structural parameters together with the space groups and tolerance factors are listed in Table III.

Possible changes in the oxidation states of Co and Ir due to the substitution of $\mathrm{La}$ by $\mathrm{Sr}$ should be reflected in the transition-metal oxygen bond lengths along the series. The average bond lengths of $\mathrm{Co}-\mathrm{O}$ and $\mathrm{Ir}-\mathrm{O}$ were calculated using the software DIAMOND (Ref. 25) and are shown in Figs. 1(a) and 1(b). The decreasing bond lengths with increasing

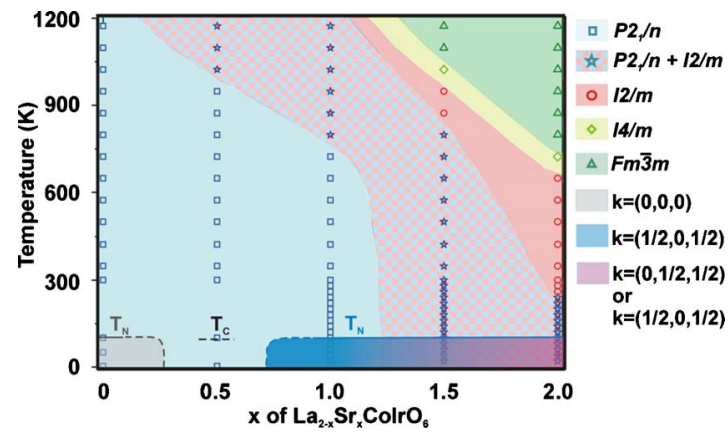

FIG. 2. (Color online) Composition and temperature dependence in the phase diagram of $\mathrm{La}_{2-x} \mathrm{Sr}_{x} \mathrm{CoIrO}_{6}$ together with the three different types of magnetic structures at low temperature. The symbols denote the experimentally obtained data. 
TABLE IV. Bond valence sums calculated from Co-O and Ir-O at room temperature.

\begin{tabular}{lcccc}
\hline \hline$x$ & $\mathrm{BVS}\left[r_{0}\left(\mathrm{Co}^{2+}-\mathrm{O}^{2-}\right)\right]$ & $\mathrm{BVS}\left[r_{0}\left(\mathrm{Co}^{3+}-\mathrm{O}^{2-}\right)\right]$ & $\mathrm{BVS}\left[r_{0}\left(\mathrm{Ir}^{4+}-\mathrm{O}^{2-}\right)\right]$ & $\mathrm{BVS}\left[r_{0}\left(\mathrm{Ir}^{5+}-\mathrm{O}^{2-}\right)\right]$ \\
\hline 0 & 2.22 & 2.26 & 3.98 & 4.52 \\
0.5 & 2.20 & 2.24 & 4.30 & 4.88 \\
1.0 & 2.30 & 2.34 & 4.54 & 5.14 \\
$1.5 P 2_{1} / n$ & 2.26 & 2.30 & 4.76 & 5.38 \\
$I 2 / m$ & 2.70 & 2.76 & 4.66 & 5.28 \\
2.0 & 2.68 & 2.74 & 4.80 & 5.42 \\
\hline \hline
\end{tabular}

Sr content are an indication for a transition in the oxidation state of $\mathrm{Co}$ and Ir. Only minor changes in the Co-O bond length with Sr-content $x$ are observed in space group $P 2_{1} / n$, but a significantly shorter $\mathrm{Co}-\mathrm{O}$ bond length is observed in the phase with space group $I 2 / \mathrm{m}$. In contrast the Ir-O bond length becomes considerably shorter with increasing $\mathrm{Sr}$ content within the stability range of the $P 2_{1} / n$ phase. This is a strong indication that La substitution by $\mathrm{Sr}$ results first (up to about $x=1.5)$ in a higher oxidation state of Ir whereas a higher oxidation state of Co is accompanied by the transition from the $P 2_{1} / n$ phase into the $I 2 / \mathrm{m}$ phase for high levels of substitution $(x \geq 1.5)$. In order to interpret the above indications BVSs for Co and Ir are calculated using the equation introduced by Brown and Altermatt. ${ }^{26}$ The reference bond lengths $r_{0}(M-\mathrm{O})$ are taken from Ref. 27 and the results are shown in Table IV. The values of the BVS confirm the observed trend of the bond lengths. Whereas regarding each compound, the bond valence sums for $\mathrm{Co}$ are similar for $r_{0}\left(\mathrm{Co}^{2+}-\mathrm{O}^{2-}\right)$ and $r_{0}\left(\mathrm{Co}^{3+}-\mathrm{O}^{2-}\right)$ cases, the sums for Ir are different for $r_{0}\left(\mathrm{Ir}^{4+}-\mathrm{O}^{2-}\right)$ and $r_{0}\left(\mathrm{Ir}^{5+}-\mathrm{O}^{2-}\right)$ cases, nevertheless they show the same trend with increasing $\mathrm{Sr}$ content. It is evident that there are only minor changes in the $\mathrm{Co}^{2+}$ state in the $P 2_{1} / n$ space group that abruptly changes into a $\mathrm{Co}^{3+}$ state in the $I 2 / m$ space group for $x \geq 1.5$, whereas for Ir the transition $\mathrm{Ir}^{4+} \rightarrow \mathrm{Ir}^{5+}$ takes place gradually within the $P 2_{1} / n$ space group and there are only minor changes in the $I 2 / \mathrm{m}$ space group [for the reference bond length $r_{0}\left(\mathrm{Ir}^{5+}-\mathrm{O}^{2-}\right)$ even a partial amount of $\operatorname{Ir}^{6+}$ is predicted for higher $\mathrm{Sr}$ content, but it may be better approximated to $\left.\mathrm{Ir}^{5+}\right]$. Therefore a partial transition in the oxidation state of $\mathrm{Co}$ and $\mathrm{Ir}$ from $\mathrm{Co}^{2+} / \mathrm{LS}-\mathrm{Ir}^{4+} \rightarrow \mathrm{HS}-\mathrm{Co}^{3+} / \mathrm{LS}^{2}-\mathrm{Ir}^{5+}$ could be concluded. Almost the same trend can be observed in the composition dependence of the Bader charges, which are shown in Table $\mathrm{V}$. The only difference being the nonpronounced increase in the Bader charge of Co in the space group $I 2 / m$ for $x=1.5$. However, Rietveld refinements reveal a high degree of cation-site disorder between $B$ and $B^{\prime}$ sites. The site disorder increases from $y=5.4 \%$ to $13.6 \%$, indicating an increasing miscibility of Co and Ir ions [Fig. 1(b)]. The difference in ionic radii between $\mathrm{Co}^{2+}(0.745 \AA)$ and $\operatorname{Ir}^{4+}(0.625 \AA)$ is $0.12 \AA$ whereas between $\mathrm{Co}^{3+}(0.61 \AA)$ and $\operatorname{Ir}^{5+}(0.57 \AA)$ it is only $0.04 \AA .{ }^{28}$ The increase in the site disorder for high values of $x$ further supports the assumption of the proposed transition of the oxidation states. However, the rather short Co-O bond length in the $I 2 / \mathrm{m}$ phase can partially result from the relative high admixture of Ir on the Co site and the higher weight of the shorter Ir-O bonds.

\section{B. Temperature dependence of crystal structures}

Rietveld refinements were performed for all five compositions, based on diffraction patterns from 3 to $1173 \mathrm{~K}$. Sequences of phases with different crystal structures have been observed with respect to both composition $x$ and temperature $T$ as summarized in Fig. 2. While the $P 2_{1} / n$ phase for $\mathrm{La}_{2} \mathrm{CoIrO}_{6}(x=0)$ remains stable up to $1173 \mathrm{~K}$, a substitution of $25 \% \mathrm{La}$ by $\mathrm{Sr}(x=0.5)$ is sufficient for a temperatureinduced phase transition from the $P 2_{1} / n$ phase into a twophase coexistence region above $923 \mathrm{~K}$. A further increase in the $\mathrm{Sr}$ content reduces the transition temperature into the two-phase region, for $x=1$ down to $750 \mathrm{~K}$ and for higher $\mathrm{Sr}$ contents down to the lowest temperatures. For $x=1.5$ and 2 three successive phase transitions are observed during heating: from the two-phase region the primitive monoclinic phase disappears for $x=2$ already at $260 \mathrm{~K}$ below room temperature. The phase transition from $P 2_{1} / n$ into $I 2 / m$ is of first order with a broad coexistence range of both phases. At elevated temperature a further phase transition $I 2 / \mathrm{m} \leftrightarrow I 4 / \mathrm{m}$ takes place at around $973 \mathrm{~K}$ for $x=1.5$ and at around $625 \mathrm{~K}$ for $x=2$. This phase transition is of second order with no discontinuity in volume (Fig. 3). This tetragonal bodycentered phase has only a rather small stability window and at higher temperatures a further phase transition of second order from $I 4 / m \leftrightarrow F m \overline{3} m$ is observed at around $1073 \mathrm{~K}$ for $x=1.5$ and $700 \mathrm{~K}$ for $x=2$. The temperature dependences of lattice parameters are shown in Figs. 4(a)-4(c) for selected compounds over characteristic temperature ranges. From the phase diagram it becomes evident that the symmetry of the

TABLE V. Bader charges calculated from charge densities associated with the lattice parameters at 3 $\mathrm{K}$.

\begin{tabular}{lccccccc}
\hline \hline$x$ & 0 & 0.5 & 1.0 & $1.5 P 2_{1} / n$ & $1.5 I 2 / m$ & $2.0 P 2_{1} / n$ & $2.0 I 2 / m$ \\
\hline Co & 1.37 & 1.37 & 1.42 & 1.42 & 1.47 & 1.72 & 1.69 \\
Ir & 1.69 & 1.82 & 1.91 & 1.98 & 1.98 & 1.98 & 2.01 \\
\hline \hline
\end{tabular}




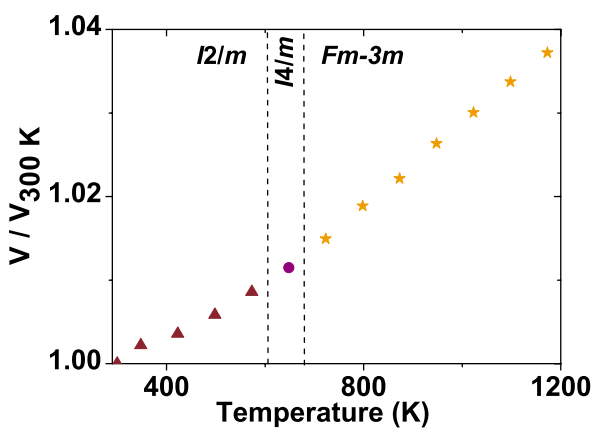

FIG. 3. (Color online) Temperature dependence of the normalized volume of $\mathrm{Sr}_{2} \mathrm{CoIrO}_{6}$.

crystal structure increases with increasing average size of the $A$-site cations at constant temperature, in agreement with other systems with double perovskite structure. ${ }^{24}$ Depending on temperature and composition the following sequence of phase transitions is observed: $P 2_{1} / n \leftrightarrow P 2_{1} / n$ $+I 2 / m \leftrightarrow I 2 / m \leftrightarrow I 4 / m \leftrightarrow F m \overline{3} m$. This sequence of structural phase transitions was already reported for $\mathrm{Sr}_{2} \mathrm{MnTeO}_{6}$,
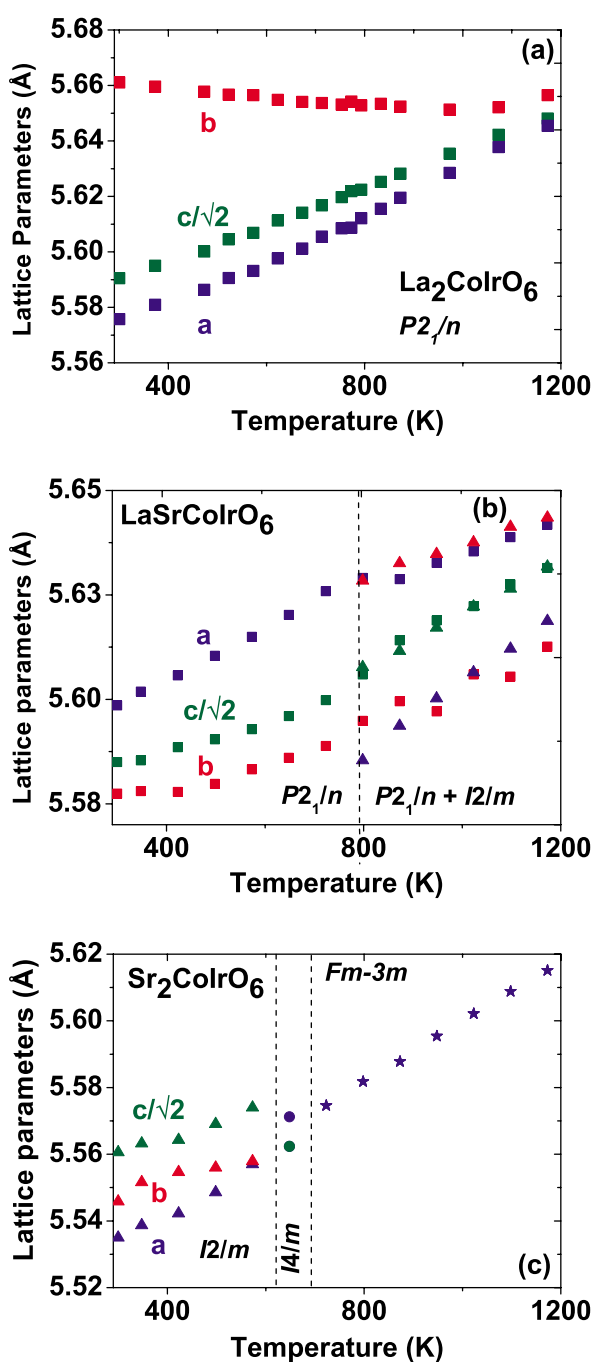

FIG. 4. (Color online) Temperature dependence of the lattice parameters for (a) $x=0$, (b) $x=1$, and (c) $x=2$.
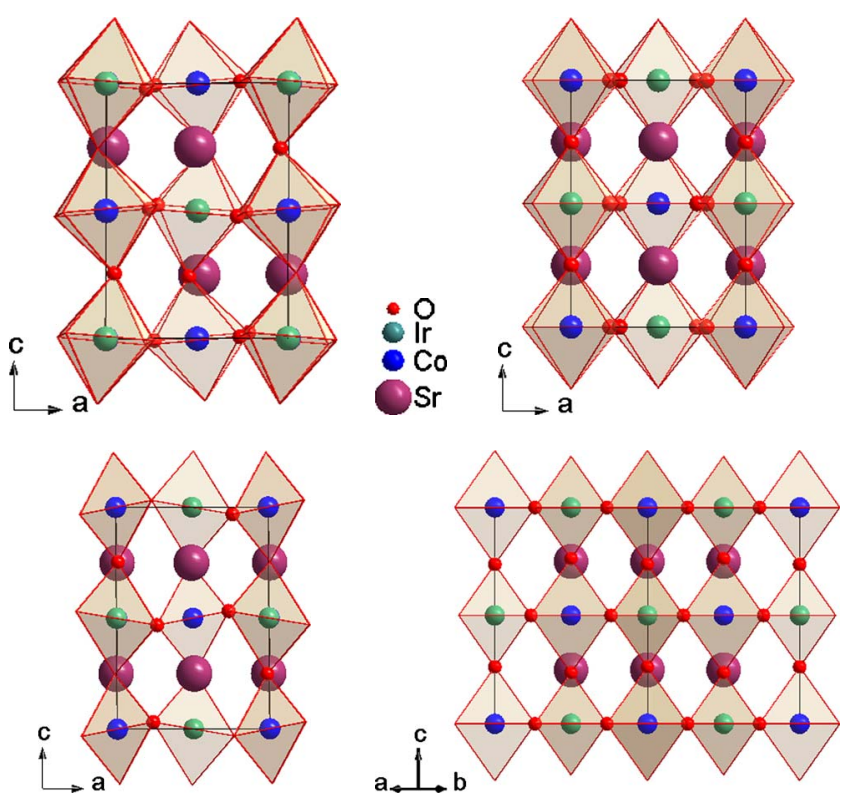

FIG. 5. (Color online) Unit cells of the modifications of $\mathrm{Sr}_{2} \mathrm{CoIrO}_{6}$ in different space groups: $P 2_{1} / n\left(a^{-} a^{-} c^{+}\right)$(top left), $I 2 / m\left(a^{0} b^{-} b^{-}\right)$(top right), I4/m $\left(a^{0} a^{0} c^{-}\right)$(bottom left), and $F m \overline{3} m\left(a^{0} a^{0} a^{0}\right)$ (bottom right).

where the first-order nature of the phase transition $P 2_{1} / n \leftrightarrow I 2 / m$ was confirmed by the existence of a thermal hysteresis in differential scanning calorimetry measurements. ${ }^{29}$ But the stability window of the coexistence of the two monoclinic phases in case of $\mathrm{Sr}_{2} \mathrm{MnTeO}_{6}$ is only $50 \mathrm{~K}$ between 250 and $300 \mathrm{~K}$, thus the coexistence does not extend to lower temperatures as in the case for $x=1.5$ and 2 . Such a coexistence of two monoclinic phases at lower temperatures is found in the double perovskite system $\mathrm{Ca}_{x} \mathrm{Sr}_{2-x} \mathrm{FeReO}_{6}$ for $1 \leq x \leq 2$. But in that case both of the monoclinic phases belong to the same space group $P 2_{1} / n$ (Refs. 30 and 31). Furthermore the factors that influence this particular phase transition with coexistence of two monoclinic phases are also different for both of the systems. Whereas for $x=1.5$ of $\mathrm{La}_{2-x} \mathrm{Sr}_{x} \mathrm{CoIrO}_{6}$ below room temperature, the phase coexistence (transition) is accompanied by a sudden increase in the oxidation state of Co in the $I 2 / \mathrm{m}$ phase due to hole doping through $A$-site cation, the $A$-site cation in $\mathrm{Ca}_{x} \mathrm{Sr}_{2-x} \mathrm{FeReO}_{6}(1 \leq x \leq 2)$ has only the size degree of freedom, therefore the phase coexistence (transition) is accompanied by a charge transfer within $B$ and $B^{\prime}$ sites induced by structural distortions. ${ }^{31}$ The crystal structures corresponding to the different phases of $\mathrm{Sr}_{2} \mathrm{CoIrO}_{6}$ are shown in Fig. 5 for a view along the monoclinic $b$ axis.

\section{Temperature and field dependence of magnetizations}

The temperature dependence of magnetization of $\mathrm{La}_{2-x} \mathrm{Sr}_{x} \mathrm{CoIrO}_{6}$ in an external field of $0.05 \mathrm{~T}$ reveals magnetic order below $100 \mathrm{~K}$ for all compositions, see Fig. 6, but the specific magnetization is reduced about almost two orders of magnitude with increasing substitution of La by Sr. The differences between magnetizations in FC and ZFC modes indicate the presence of ferromagnetic components, 


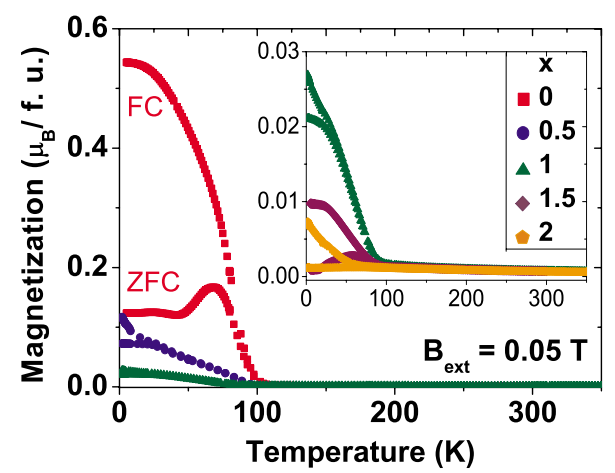

FIG. 6. (Color online) Temperature dependence of magnetization for $\mathrm{La}_{2-x} \mathrm{Sr}_{x} \mathrm{CoIrO}_{6}$.

confirmed by the hysteresis loops in Fig. 7. Curie temperatures were determined using the linear extrapolation method and decrease from $90 \mathrm{~K}$ for $x=0-1.5$ to $70 \mathrm{~K}$ for $x=2$ (Table VI). The paramagnetic Curie-Weiss temperatures $\theta$, determined from the Curie-Weiss fits are negative along the whole series, indicating dominant antiferromagnetic interactions, which become stronger with increasing $\mathrm{Sr}$ content (Table VI). The low values for the ferromagnetic components, determined as the spontaneous magnetizations by linear extrapolation of the high-field dependence of magnetization to zero field (Table VI) and the strong antiferromagnetic interactions indicate NCM with ferromagnetic and antiferromagnetic components of the ordered magnetic moments. Magnetizations are not saturated at $7 \mathrm{~T}$, further supporting the proposed NCM for the whole series. Another characteristic feature of the hysteresis loops for $x=0$ and 1 is their shape, which might reflect an external-field-induced magnetic phase transition as already found in many compounds with NCM, due to magnetic anisotropy, that results in minima in the angle- (of the magnetic moments) dependent energy curves. ${ }^{32}$ Regarding the double perovskites, investigations on such magnetic phase transitions are rare and known only for a few compounds such as $\mathrm{Ca}_{2} \mathrm{FeReO}_{6}$ (Ref. 31), $\mathrm{Sr}_{2} \mathrm{NiReO}_{6}$ (Ref. 33), and $\mathrm{Sr}_{2} \mathrm{RuGdO}_{6}$ (Ref. 34). But the condition under which this transition occurs is different. For $\mathrm{Ca}_{2} \mathrm{FeReO}_{6}$, mainly a metal-insulator transition from metallic FM to an insulating FM (actually coexisting below the transition temperature) induces the spin reorientation, ${ }^{35}$ where the insulating FM transits gradually to the metallic FM at higher external magnetic fields below the transition temperature, ${ }^{31}$

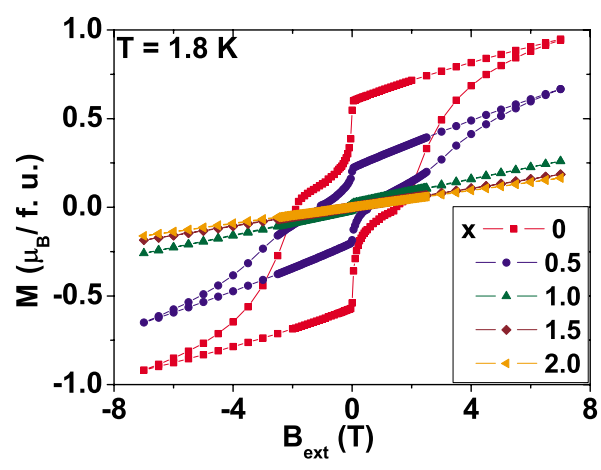

FIG. 7. (Color online) Field dependence of magnetization for $\mathrm{La}_{2-x} \mathrm{Sr}_{x} \mathrm{CoIrO}_{6}$.

whereas for $\mathrm{Sr}_{2} \mathrm{NiReO}_{6}$ and $\mathrm{Sr}_{2} \mathrm{RuGdO}_{6}$ the external-fieldinduced magnetic phase transition takes place in the AFM phase with a small ferromagnetic component. The fieldinduced magnetic phase transition in $\mathrm{La}_{2} \mathrm{CoIrO}_{6}$ and $\mathrm{La}_{1.5} \mathrm{Sr}_{0.5} \mathrm{CoIrO}_{6}$ can be therefore comparable with $\mathrm{Sr}_{2} \mathrm{NiReO}_{6}$ and $\mathrm{Sr}_{2} \mathrm{RuGdO}_{6}$. The effective magnetic moments $\mu_{\text {eff }}$ were calculated from the Curie constants and are compared with the spin-only values of $\mathrm{Co}$, based on the assumption of $\mathrm{HS}_{-} \mathrm{Co}^{2+}$ for $x=0$ and $\mathrm{HS}-\mathrm{Co}^{3+}$ for $x=2$. The experimentally determined values for $\mu_{\mathrm{eff}}$ are always larger than the calculated spin-only values especially for the $\mathrm{Co}^{2+}$ case (Table VI), consistent with other double perovskites containing $\mathrm{Co}^{2+}$ (Ref. 33). This discrepancy indicates significant contributions to the paramagnetic moments from Ir and/or orbital momentum.

\section{Magnetic structure}

Magnetic superstructure Bragg reflections were observed in NPD patterns recorded at temperatures below $100 \mathrm{~K}$. This reveals long-range antiferromagnetic order in $\mathrm{La}_{2-x} \mathrm{Sr}_{x} \mathrm{CoIrO}_{6}$. The positions of the magnetic reflections depend on composition and were indexed with the propagation vectors $k=(0,0,0)$ for $\mathrm{La}_{2} \mathrm{CoIrO}_{6}(x=0), k=(1 / 2,0,1 / 2)$ for $x=1$, and $k=(0,1 / 2,1 / 2)$ or $k=(1 / 2,0,1 / 2)$ for $x=1.5$ and 2 , respectively. For $x=0.5$ only very weak magnetic reflections were observed that could not be indexed unambiguously. The refined magnetic moment at the Co site varies from $\left(\mu_{\mathrm{x}}, 0, \mu_{\mathrm{z}}\right)$ with $\mu_{\mathrm{x}}=-0.7(1) \mu_{\mathrm{B}}, \mu_{\mathrm{z}}=1.5(1) \mu_{\mathrm{B}}$, and $|\mu|$ $=1.7(1) \mu_{\mathrm{B}}$ for $x=0$ to $\left(\mu_{\mathrm{x}}, 0, \mu_{\mathrm{z}}\right)$ with $\mu_{\mathrm{x}}=1.2(1) \mu_{\mathrm{B}}, \mu_{\mathrm{z}}$

TABLE VI. Ferromagnetic Curie-temperature $T_{\mathrm{C}}$, paramagnetic Curie-Weiss temperature $\theta$, spontaneous magnetization $M_{\mathrm{S}}$, experimentally determined and calculated effective magnetic moments $\mu_{\text {eff }}$ and $\mu_{\text {eff }}(\mathrm{Co}-$ spin only).

\begin{tabular}{lccccc}
\hline \hline & $\begin{array}{c}\mu_{\text {eff }} \\
\left(\mu_{\mathrm{B}} / \text { f.u. }\right)\end{array}$ & $\begin{array}{c}\mu_{\text {eff }}(\text { Co-spin only }) \\
\left(\mu_{\mathrm{B}} / \text { f.u. }\right)\end{array}$ & $\begin{array}{c}\Theta \\
(\mathrm{K})\end{array}$ & $\begin{array}{c}M_{\mathrm{S}} \\
\left(\mu_{\mathrm{B}} / \text { f.u. }\right)\end{array}$ & $\begin{array}{c}T_{\mathrm{C}}(\text { linear extrapolation }) \\
(\mathrm{K})\end{array}$ \\
\hline 0 & $4.7(1)$ & $3.88\left(\mathrm{Co}^{2+}\right)$ & $-13.8(6)$ & 0.7 & 95 \\
0.5 & $5.0(1)$ & & $-55.1(8)$ & 0.15 & 91 \\
1 & $5.1(0)$ & & $-94.9(1)$ & 0.03 & 89 \\
1.5 & $5.0(0)$ & & $-103.0(5)$ & 0.015 & 91 \\
2 & $5.1(1)$ & $4.90\left(\mathrm{Co}^{3+}\right)$ & $-138.8(0)$ & 0.005 & 70 \\
\hline \hline
\end{tabular}



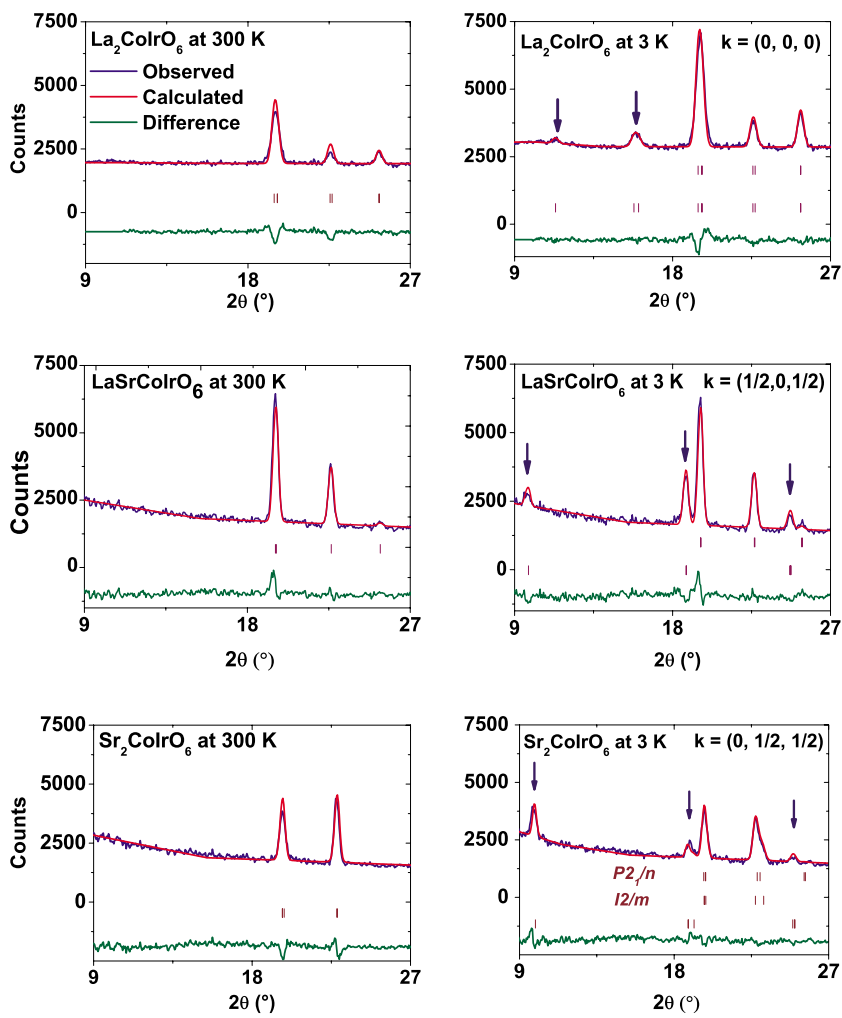

FIG. 8. (Color online) Observed, calculated, and difference Rietveld profiles for $\mathrm{La}_{2} \mathrm{CoIrO}_{6}, \mathrm{LaSrCoIrO}_{6}$, and $\mathrm{Sr}_{2} \mathrm{CoIrO}_{6}$ at 3 $\mathrm{K}$. Low-angle region of the profile for $\mathrm{La}_{2} \mathrm{CoIrO}_{6}, \mathrm{LaSrCoIrO}_{6}$, and $\mathrm{Sr}_{2} \mathrm{CoIrO}_{6}$ at 300 and $3 \mathrm{~K}$, where prominent magnetic superstructure Bragg reflections were present. Reflection markers correspond to the positions of Bragg reflections of structural (top) and magnetic (bottom) contributions.

$=2.3(1) \mu_{\mathrm{B}}$ and $|\mu|=2.6(1) \mu_{\mathrm{B}}$ for $x=1$ and $\left(\mu_{\mathrm{x}}, 0, \mu_{\mathrm{z}}\right)$ with $\mu_{\mathrm{x}}=1.6(1) \mu_{\mathrm{B}}, \mu_{\mathrm{z}}=2.1(1) \mu_{\mathrm{B}}$, and $|\mu|=2.6(1) \mu_{\mathrm{B}}$ for $x=2$ [or for the $k=(1 / 2,0,1 / 2)$ case: $\left(0,0, \mu_{\mathrm{z}}\right)$ with $\mu_{\mathrm{z}}=2.6(1) \mu_{\mathrm{B}}$ and $\left.|\mu|=2.6(1) \mu_{\mathrm{B}}\right]$. The observed and calculated diffraction profiles together with the magnetic reflections are shown in Fig. 8 and the three types of antiferromagnetic structures in Fig. 9.
Four ambiguities remain in the magnetic structures derived from NPD. (1) The ferromagnetic component could not be extracted quantitatively from NPD, because the corresponding intensities are too low in comparison with the dominant nuclear contributions. Nevertheless, an analysis of magnetic symmetries reveals that the ferromagnetic component should be along the [010] direction (AFM component on the $x z$ plane). (2) For $x=1$ the $k=(1 / 2,0,1 / 2)$ AFM structure is preferred against the $k=(0,1 / 2,1 / 2)$ AFM structure, based on a better agreement of calculated and observed intensities of the magnetic Bragg reflections, For $x=1.5$ and 2 no major differences exist between $k=(0,1 / 2,1 / 2)$ and $k$ $=(1 / 2,0,1 / 2)$ types of magnetic structures. (3) Any contribution from ordered magnetic moments on the Ir site is too small to be detected. The magnetic moments were therefore refined only at Co sites. (4) As the Co sublattices are almost identical in the coexisting primitive and I-centered monoclinic modifications, the magnetic contributions for $x=1.5$ and 2 cannot be assigned to one of these phases distinctly, but the magnetic arrangement (see Fig. 9 on the right-hand side) is unambiguous.

In all three magnetic structures alternating ferromagnetic planes exist, but they are different in all the structures. A qualitative explanation is based on the magnetic superexchange interactions. The geometries of the underlying exchange paths deviate from those in idealized intersecting fcc sublattices of $\mathrm{Co}$ and Ir ions due to the monoclinic distortions. The corresponding face-centered pseudocell for $\mathrm{Co}$ is shown in Fig. 9 on the left. According to Ref. 36 the $k$ $=(0,0,0)$ magnetic structure results from a strong nearestneighbor antiferromagnetic Co-O-O-Co interaction within the Co sublattice in competition with the $180^{\circ}$ antiferromagnetic next-nearest-neighbor $\sigma$ Co-O-Ir-O-Co interaction between the Co sublattices through the vacant $e_{\mathrm{g}}$ orbitals of Ir. However, in this case, the Co-O-Ir angle is only $152.5^{\circ}$ due to the distorted crystal structure and, therefore, this interaction is weaker. This scenario is almost realized in $\mathrm{La}_{2} \mathrm{CoIrO}_{6}$, except for the small ferromagnetic component along the $b$ direction, resulting from the $180^{\circ}\left(152.5^{\circ}\right.$ in this case) CoO-Ir ferromagnetic interaction (Ir has five $t_{2 \mathrm{~g}}$ electrons) (Ref. 37) between Co and Ir sublattices, which is frustrated and probably responsible for a Dzyaloshinski-Moriya interaction.
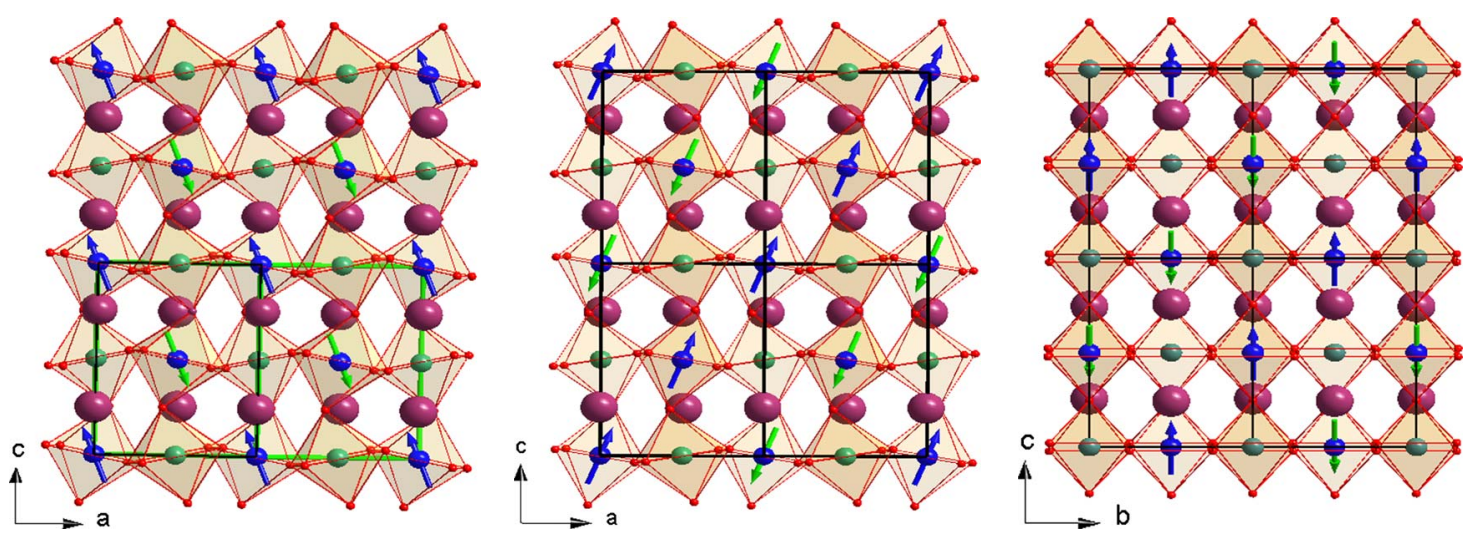

FIG. 9. (Color online) Three different types of magnetic structures with $k=(0,0,0)$ for $x=0$ viewed along the $b$ axis (left), $k$ $=(1 / 2,0,1 / 2)$ for $x=1$ along the $b$ axis (middle), and $(0,1 / 2,1 / 2)$ for $x=2$ along the $a$ axis (right). Magnetic unit cells (black) and the distorted face-centered lattice of Co (green online, gray in print) are also shown. 

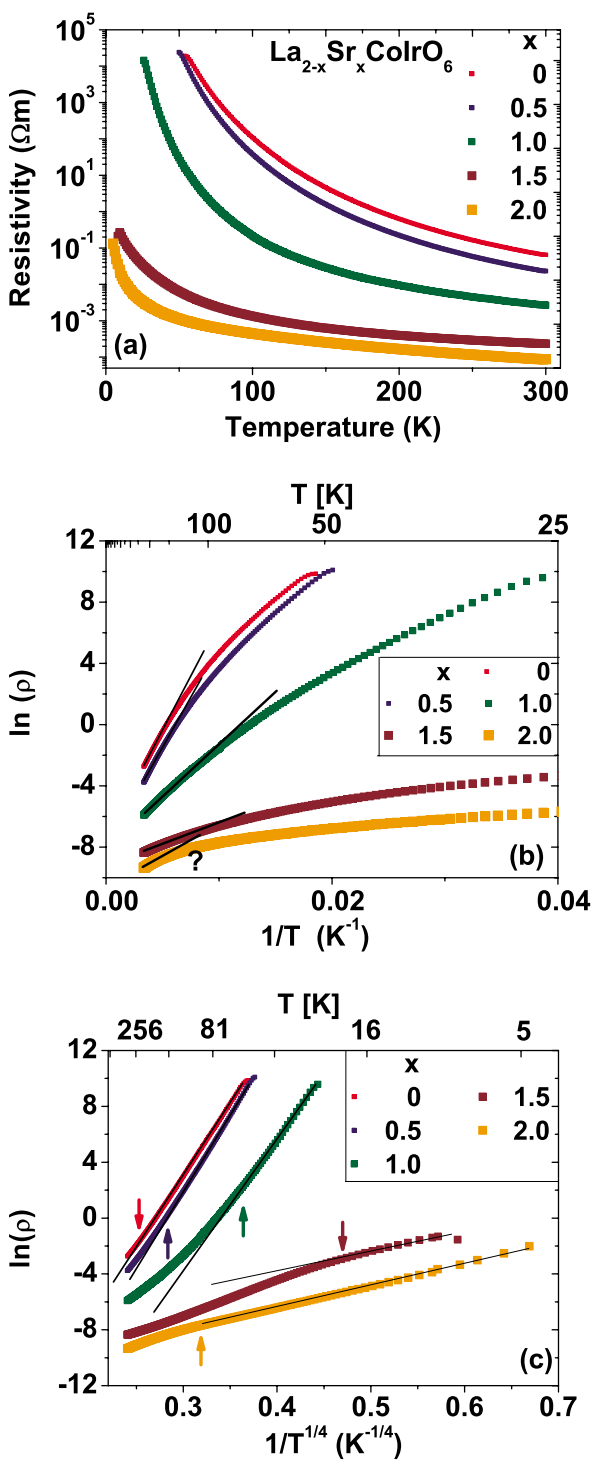

FIG. 10. (Color online) (a) Temperature dependence of resistivity for $x=0,0.5$, and 1 in logarithmic scale, (b) $\ln (\rho)$ vs $1 / T$. A linear fit describes the high-temperature behavior for $x \leq 1.5$, but fails for $x=2$ and (c) $\ln (\rho)$ vs $1 / T^{1 / 4}$. The arrows mark the maximum temperature, up to which a linear dependence is well obeyed.

Similar interactions are proposed for $\mathrm{La}_{1+x} \mathrm{Ca}_{1-x} \mathrm{CoRuO}_{6}$, where both $B$ and $B^{\prime}$ sites are occupied with magnetic ions. ${ }^{38}$ For increasing $x$ the Co-O-Ir bond angle along the $\sigma$ Co-OIr-O-Co path increases up to $166.3^{\circ}$ for $x=2$, and this coupling becomes dominant for $x=1$ and 2 , resulting either in the $k=(1 / 2,0,1 / 2)$ or the $k=(0,1 / 2,1 / 2)$ type magnetic structure, which differs only in the strength of the secondary $90^{\circ}$ Co-O-Ir-O-Co exchange paths. ${ }^{39}$

\section{E. Transport properties}

Resistivity measurements reveal a nonmetal-like behavior for the whole series of samples, but the resistivity is reduced by up to three orders of magnitude at room temperature when $\mathrm{La}$ is replaced by $\mathrm{Sr}$ on the $A$ site. Three different representations of the temperature dependences of resistivity are shown in Figs. 10(a)-10(c) to discuss the underlying transport mechanism: a linear dependence of $\ln \rho$ vs $1 / T$ indicates a simple thermal activation of electronic transport in contrast to a characteristic linear dependence of $\ln \rho$ vs $1 / T^{1 / 4}$ for a variable-range hopping (VRH) model as introduced by Mott for disordered systems. ${ }^{40}$ Both models are inadequate to describe the temperature dependence over the whole investigated temperature range, but appear suitable either in the lower or higher temperature regime. Therefore, a combination of both behaviors [Eq. (1)] was applied to describe the experimental data over the whole temperature range

$$
\frac{1}{\rho}=\frac{1}{\rho_{01}} e^{-\left(E_{\mathrm{G}} / 2 k_{B} T\right)}+\frac{1}{\rho_{02}} e^{-\left(T_{0} / T\right)^{1 / 4}} .
$$

Accordingly, the activated (band) gaps $E_{\mathrm{G}}$ in the lowtemperature regions vary from $0.26 \mathrm{eV}$ for $x=0$ and 0.5 over $0.12 \mathrm{eV}$ for $x=1$ to $0.05 \mathrm{eV}$ for $x=1.5$. For $x=2$ the VRH mechanism dominates up to about $100 \mathrm{~K}$, but the thermalactivation model does not give a proper fit at higher temperatures. This peculiar situation might be caused by the high degree of $B / B^{\prime}$-site cation disorder of $15 \%$ for $\mathrm{Sr}_{2} \mathrm{CoIrO}_{6}$. Assuming a band gap in accordance with the trend of this system, $E_{\mathrm{G}} \leq 0.05 \mathrm{eV}$ is expected for $x=2$ and means that the distribution width of localized states within the gap approaches the band gap and this will result in a different mechanism for electron activation. ${ }^{41}$

The substitution of La by $\mathrm{Sr}$ has mainly two effects on the transport properties, which are closely related to the two degrees of freedom mentioned in the introduction: On one hand the number of electrons at the $B$ site is reduced (hole doping) which affects the band filling and reduces the electronelectron Coulomb repulsion. In the $P 2_{1} / n$ space group $(x$ $\leq 1.5$ ), in agreement with the bond valence analysis, the holes are introduced predominantly in the $t_{2 \mathrm{~g}}$ bands of $\mathrm{Ir}$ $\left(\mathrm{Ir}^{4+} \rightarrow \mathrm{Ir}^{5+}\right)$, whereas in the $I 2 / m$ space group $(x \geq 1.5)$ they are predominantly introduced in the $3 d$ bands of Co. On the other hand the average Co-O-Ir bond angle, determined from Rietveld refinements, increases from $152.5^{\circ}$ for $x=0$ to $166.3^{\circ}$ for $x=2$ and means a broadening of the bandwidth. Both effects result in an increase in the electronic conductivity as observed. The temperature dependence of resistivity was also measured in an external magnetic field of $B=1 \mathrm{~T}$. No significant magnetoresistance effect was detected nor any anomalies at the magnetic-ordering temperatures. Similar systematic investigations of the influence of the $A$-site cation on the transport properties of double perovskites are reported for $\mathrm{La}_{1+x} \mathrm{Sr}_{1-x} \mathrm{CoRuO}_{6} \quad(-0.5 \leq x \leq 0.25) \quad$ (Ref. 42) $\left[\mathrm{La}_{2-x} \mathrm{Sr}_{x} \mathrm{CoRuO}_{6} \quad(0 \leq x \leq 2) \quad\right.$ (Ref. 43)] and $\mathrm{La}_{1+x} \mathrm{Ca}_{1-x} \mathrm{CoRuO}_{6}(-0.25 \leq x \leq 0.25)$ (Ref. 38). But in contrast to an increase in the conductivity throughout the series as in the present case by $\mathrm{Sr}$ substitution, the conductivity is increased first by the hole doping into $\mathrm{Ru} 4 t_{2 \mathrm{~g}}$ but as the ratio of $\mathrm{Ru} 3 t_{2 \mathrm{~g}}$ increases, the conductivity decreases and has a minimum for $\mathrm{LaSrCoRuO}_{6}$ with $\mathrm{Ru} 3 t_{2 \mathrm{~g}}$ configuration. Further doping introduces holes into $t_{2 \mathrm{~g}}$ bands of $\mathrm{Co}$ and the conductivity increases once again. 

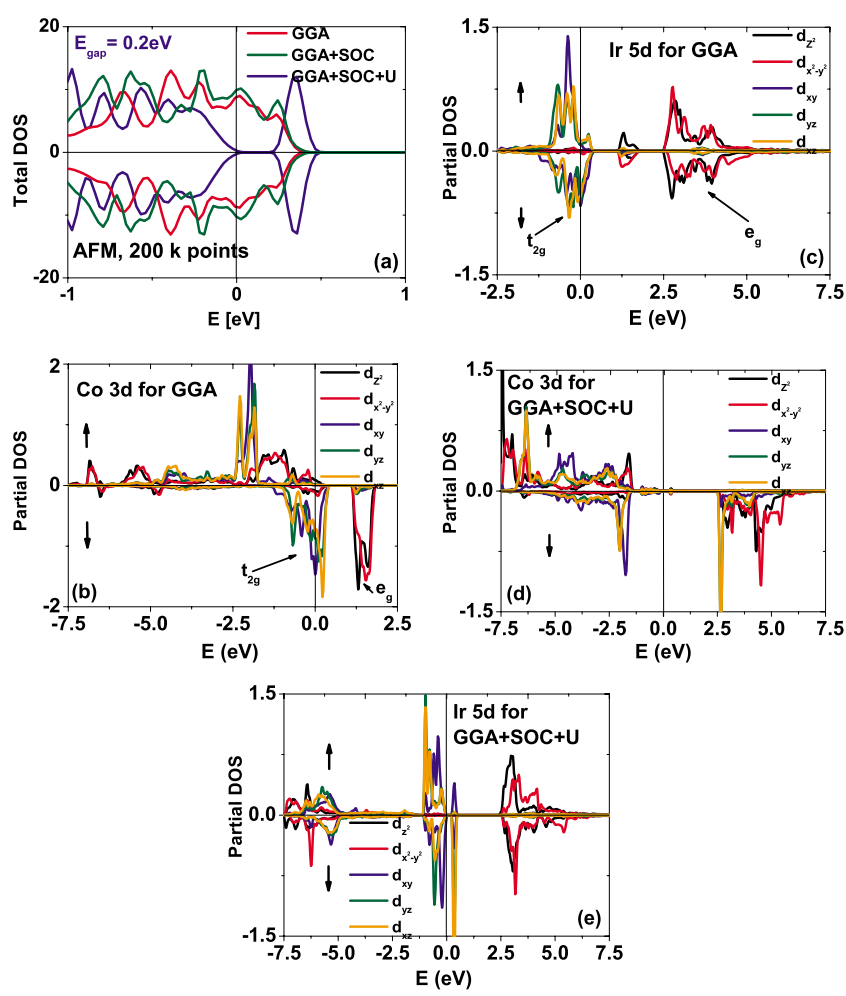

FIG. 11. (Color online) (a) Total DOS of AFM for $x=0$ within GGA, GGA+SOC, and GGA + SOC $+U$ (top). (b) Partial DOS of Co $3 d$ bands and (c) Ir $5 d$ bands for GGA (middle). (d) Partial DOS of Co $3 d$ bands and (e) Ir $5 d$ bands for GGA $+\mathrm{SOC}+U$ (bottom). Note that for $x=0$ there are two independent sites for Co and Ir each with opposite magnetic moments. Partial DOS in (b), (d) and (c), (e) correspond to only one Co (Co 2) and one Ir (Ir 2).

\section{F. Electronic structures}

The electronic structure calculations are discussed in four parts, addressing (i) the density of states (DOS), (ii) a comparison of total energies for different configurations of $x=0$, (iii) the oxidation state of $\mathrm{Co}$ and $\mathrm{Ir}$, and (iv) the magnetic moments.

(i) The calculated DOS for $x=0$ of the experimentally found $k=(0,0,0)$ AFM structure is shown in Fig. 11(a) for the GGA case. It is evident from the total DOS and the calculated occupancies of the $d$ bands, that $x=0$ has a metallic ground state with predominant $\mathrm{HS}-\mathrm{Co}^{2+}$ and $\mathrm{LS}-\mathrm{Ir}^{4+}$ in contrast to the experimental finding of an insulator with a band gap of $0.26 \mathrm{eV}$. Additionally the 1-decomposed partial DOS of Co $3 d(\mathrm{Co} 2)$ and Ir $5 d(\operatorname{Ir} 2)$ for $x=0$, projected on the local coordinate system along the $B / B^{\prime}-\mathrm{O}$ bond direction in the oxygen octahedra are also shown in Figs. 11(b) and 11(c). From the partial DOS of Co $3 d$ and Ir $5 d$ it is evident that the three $t_{2 \mathrm{~g}}$ bands of Co-3d are similarly occupied (neglecting the additional break down of the $t_{2 \mathrm{~g}}$ degeneracy due to the monoclinic distortion: $d_{\mathrm{xy}}, d_{\mathrm{yz}}$, and $d_{\mathrm{xz}}$ ) by the two spin-down electrons per Co atom and the three $t_{2 \mathrm{~g}}$ bands of Ir $5 d$ are filled by 2.5 spin-up/-down electrons per Ir atom and thus a metallic state is obtained. The experimental nonmetal behavior with a band gap should be attributed to strong electron-electron onsite Coulomb repulsion that is badly described within the LDA/GGA approximation. A comparison

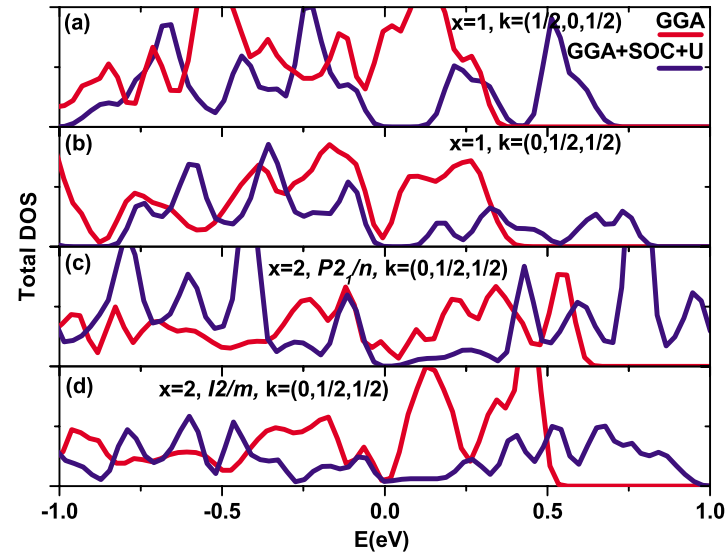

FIG. 12. (Color online) Total (spin-up) DOS of (a) $x=1 k$ $=(1 / 2,0,1 / 2), \quad$ (b) $k=(0,1 / 2,1 / 2), \quad$ (c) $\quad x=2 \quad\left(P 2_{1} / n\right) \quad k$ $=(0,1 / 2,1 / 2)$, and (d) $x=2(I 2 / m) k=(0,1 / 2,1 / 2)$ AFM configurations for the GGA (red) and $\mathrm{GGA}+\mathrm{SOC}+U(\mathrm{Co})=0.4 \mathrm{Ry}$ $+U(\mathrm{Ir})=0.2$ Ry (blue) cases. Only minor differences between GGA and GGA+SOC with respect to the DOS around the Fermi level and thus the latter are not shown for $x=1$ and 2 .

of bandwidth $W$ with typical onsite Coulomb energies $U$ is necessary to take this effect adequately into account. The approximate width $W$ of the $d$ bands could be estimated to $1.5 \mathrm{eV}$ for Co $t_{2 \mathrm{~g}}, 0.7 \mathrm{eV}$ for Co $e_{\mathrm{g}}, 2 \mathrm{eV}$ for Ir $t_{2 \mathrm{~g}}$, and 2.7 $\mathrm{eV}$ for Ir $e_{\mathrm{g}}$. Therefore, the bandwidth $W$ of both Co- $d$ and Ir- $d$ bands are small compared to (or in the case of $\operatorname{Ir} e_{\mathrm{g}}$ at most equal with) the typical onsite Coulomb repulsion energies $U$, which are around $5.44 \mathrm{eV}(0.4 \mathrm{Ry})$ and $2.72 \mathrm{eV}(0.2$ Ry) for Co and Ir, respectively. ${ }^{44}$ Therefore an onsite Coulomb repulsion $U$ has been included for the $d$ bands together with exchange parameters $J(\mathrm{Co})=0.918 \mathrm{eV}$ and $J(\mathrm{Ir})$ $=0.544 \mathrm{eV}$ via the fully localized limit (FLL) method as implemented in WIEN2K and described in Refs. 45 and 46.

The total DOS $x=0$ AFM for GGA+SOC and GGA $+\mathrm{SOC}+U$ (Co and Ir) are also shown in Fig. 11(a). It can be seen that there is not much difference in the total DOS between GGA and GGA+SOC. But as soon as $U$ for both Co and Ir were added a band gap opens up at the Fermi level which is around $0.2 \mathrm{eV}$ in fairly good agreement with the experimental finding. This is also valid for the total DOS of the FM configuration (not shown). The inclusion of $U$ shifts the two occupied spin-down " $t_{2 \mathrm{~g}}$ " orbitals around $-(U$ $-J) / 2$ and the one unoccupied $t_{2 \mathrm{~g}}$ orbital around $+(U-J) / 2$ in case of $3 d$ of a Co. In case of $5 d$ of an Ir a fairly empty spin-down $t_{2 \mathrm{~g}}$ orbital is obtained by the $+(U-J) / 2$ shift with an additional enhancement of the spin polarizations are obtained for the above reason. This splitting in the $t_{2 \mathrm{~g}}$ bands of Co $3 d$ and Ir $5 d$ results in a band gap at the Fermi level. However fractional occupancies were obtained for all the mentioned $t_{2 \mathrm{~g}}$ bands as a result of the competing hybridization and SOC as shown in Figs. 11(d) and 11(e). The " $e_{\mathrm{g}}$ " $\left(d_{\mathrm{z} 2}, d_{\mathrm{x} 2-\mathrm{y} 2}\right)$ bands also undergo the above-mentioned shifts but they are not relevant to the changes in the electronic structure at the Fermi level.

The total DOS for $x=1$ and 2 with the experimentally found $k=(1 / 2,0,1 / 2)$ and $k=(0,1 / 2,1 / 2)$ AFM structures are shown in Fig. 12. As a comparison the DOS for $x=1$ with 
TABLE VII. Total-energy differences $\Delta E$ given, relatively to the experimentally (NPD) observed $k=(0,0,0) \mathrm{AFM}$, in mRy/f.u. for FM, $k=(0,0,0) \mathrm{AFM}$, and $k=(0,1 / 2,1 / 2)$ AFM configurations for the GGA $+\mathrm{SOC}+U$ case. The total-energy differences for the relaxed structure calculations are shown in the third column, again relatively to the total energy of the relaxed $k=(0,0,0)$ AFM structure.

\begin{tabular}{lcc}
\hline \hline & $\Delta E$ & $\Delta E$ \\
$x=0$ & $\begin{array}{c}\text { (experimental } \\
\text { internal positions) }\end{array}$ & $\begin{array}{c}\text { (relaxed } \\
\text { internal positions) }\end{array}$ \\
\hline FM & -0.25 & +3.10 \\
AFM $k=(0,0,0)$ & 0.00 & 0.00 \\
AFM $k=(0,1 / 2,1 / 2)$ & +36.07 & +40.70 \\
\hline \hline
\end{tabular}

the $k=(0,1 / 2,1 / 2)$ AFM structure is also shown in Fig. 12 . For $x=1$ a band gap of around $0.1 \mathrm{eV}$ can be seen from Fig. 12 as soon as $U$ was included in the calculation, almost independent of the experimentally found $k=(1 / 2,0,1 / 2)$ and the not preferred $k=(0,1 / 2,1 / 2)$ AFM structure. For $x=2$ the total DOS is shown for the two modifications coexisting at temperatures below $220 \mathrm{~K}$. Whereas the low-temperature modification $P 2_{1} / n$ is a nonmetal with a band gap around $0.05 \mathrm{eV}$, the high-temperature modification $I 2 / \mathrm{m}$ is metallic with a rather small DOS at the Fermi level even after the inclusion of $U$. In contrast to theory, resistivity measurements reveal $x=2$ to be nonmetallic over the whole measured temperature range. However, the investigated $\mathrm{Sr}_{2} \mathrm{CoIrO}_{6}$ sample has a high degree of $B / B^{\prime}$-site disorder and is better described as $\mathrm{Sr}_{2}\left(\mathrm{Co}_{0.85} \mathrm{Ir}_{0.15}\right)\left(\mathrm{Ir}_{0.85} \mathrm{Co}_{0.15}\right) \mathrm{O}_{6}$. The nonactivationlike temperature dependence of resistivity and the apparent band gap instead of a continuous band could result from localized states at the Fermi level due to this site disorder. ${ }^{41,47}$ For the $P 2_{1} / n$ phase the calculated band gaps decrease from $0.2 \mathrm{eV}$ for $x=0$ to $0.05 \mathrm{eV}$ for $x=2$ in agreement with the observed reduction in $E_{\mathrm{G}}$ at least for $0 \leq x$ $\leq 1.5$.

(ii) As the magnitude of FM (from the magnetization measurement) and the $k=(0,0,0)$ AFM components are similar for $x=0$, the total energies of these magnetic structures together with the $k=(0,1 / 2,1 / 2)$ AFM are compared for the GGA $+\mathrm{SOC}+U$ case as shown in the second column of Table VII. All the atomic positions are taken from the structure model, refined based on NPD (experimental). For the $k=(0,1 / 2,1 / 2)$ AFM configuration the same atomic positions are taken and supercells have been built. The FM configuration has a total energy $0.25 \mathrm{mRy} / \mathrm{f}$.u. lower than the observed $k=(0,0,0)$ AFM configuration whereas for the GGA case (not shown) the total energy of the AFM configuration is lower than the FM configuration. As the inclusion of $U$ resulted in anisotropic occupancies of the $d$ orbitals, this increases the total forces (certain components of oxygen are around $100 \mathrm{mRy} / \mathrm{a} . u$.) acting on atoms and such occupations would couple to the lattice and move atoms in general positions (especially oxygen). Therefore for all three configurations, the internal atomic positions are relaxed till the forces are below $5 \mathrm{mRy} / \mathrm{a} . u$. , and these total energies are compared (Table VII, third column). The $k=(0,0,0)$ AFM configuration is energetically more favored than the FM $(+3.1 \mathrm{mRy} / \mathrm{f}$.u. $)$ one. This small difference in the total energies of the two configurations is an indication for a strong competition between ferromagnetic and antiferromagnetic interactions, and spin-orbit coupling together with electron correlations that play the key role for the observed noncollinear magnetism for $x=0$. Note that in both cases the $k$ $=(0,1 / 2,1 / 2)$ AFM configuration is energetically clearly unfavorable.

(iii) Table VIII shows the occupation of the $d$ bands of Co 2 and Ir 2 for GGA and GGA $+U+$ SOC cases. The striking difference between the two is that for $\mathrm{GGA}+\mathrm{SOC}+U$ the difference between the occupations of $x=0$ and 2 is larger than in GGA. The GGA $+\mathrm{SOC}+U$ results are interpreted as follows. For $x=0$, see Figs. 11(d) and 11(e), it is evident that the spin-up $d$ bands for Co $3 d$ are almost fully occupied while the spin-down DOS for the $e_{\mathrm{g}}\left(d_{\mathrm{z} 2}, d_{\mathrm{x} 2-\mathrm{y} 2}\right)$ bands are almost completely unoccupied. This is in good agreement with the assumed high-spin (HS) state of $\mathrm{Co}^{2+}$ and the lowspin (LS) state for $\mathrm{Ir}^{4+}$ in the simple ionic picture, neglecting hybridization effects and residual electron densities at interstitials. The proposed tendency toward $\mathrm{HS}-\mathrm{Co}^{3+} / \mathrm{Ir}^{5+}$ with increasing $\mathrm{Sr}$ content is also supported by the calculated occupation of the $d$ bands for $x=1$ and 2. The occupation of the Co $3 d$ bands changes significantly only for $x>1$ whereas changes in the occupation of the Ir $5 d$ bands are only significant for $x \leq 1$. This is in good agreement with the successive changes in $\mathrm{Co}-\mathrm{O}$ and Ir-O bond lengths, see Fig. 1.

(iv) The calculated magnetic-moment contributions from spin and orbital momentum are compared for $\mathrm{Co}$ and Ir in Table IX within GGA $+\mathrm{SOC}+U$. The calculated spin moment of Co for $x=0$ is $2.68 \mu_{\mathrm{B}}$ per Co ion $\left(2.53 \mu_{\mathrm{B}}\right.$ per Co ion for GGA) and much larger than the observed magnetic moment of $|\mu|=1.7 \mu_{\mathrm{B}}$ per Co ion (NPD). As different $3 d$ orbital occupancies for $\mathrm{Co}$ are possible within the same oxidation state and lattice parameters in $\mathrm{LDA} / \mathrm{GGA}+U,{ }^{48}$ such an or-

TABLE VIII. Calculated occupations of Co $3 d$ and Ir $5 d$ bands for the experimentally found AFM structures in units of $d$ electrons per atom.

\begin{tabular}{|c|c|c|c|c|c|c|c|c|c|c|c|c|}
\hline \multirow[b]{2}{*}{$x$} & \multicolumn{3}{|c|}{ Co GGA } & \multicolumn{3}{|c|}{$\mathrm{Co} \mathrm{GGA}+\mathrm{SOC}+U$} & \multicolumn{3}{|c|}{ Ir GGA } & \multicolumn{3}{|c|}{$\mathrm{Ir} \mathrm{GGA}+\mathrm{SOC}+U$} \\
\hline & Up & Down & Total & Up & Down & Total & Up & Down & Total & Up & Down & Total \\
\hline 0 & 4.63 & 2.11 & 6.74 & 4.69 & 2.03 & 6.72 & 2.64 & 2.11 & 4.75 & 2.70 & 2.26 & 4.96 \\
\hline 1 & 4.61 & 2.05 & 6.66 & 4.67 & 2.02 & 6.69 & 2.73 & 2.11 & 4.84 & 2.77 & 2.00 & 4.77 \\
\hline $2\left(P 2_{1} / n\right)$ & 4.56 & 1.89 & 6.45 & 4.68 & 1.65 & 6.33 & 2.75 & 1.89 & 4.64 & 2.72 & 1.91 & 4.63 \\
\hline $2(I 2 / m)$ & 4.58 & 1.91 & 6.49 & 4.67 & 1.68 & 6.35 & 2.73 & 1.93 & 4.66 & 2.76 & 1.92 & 4.68 \\
\hline
\end{tabular}


TABLE IX. Calculated spin $\left(m_{\mathrm{S}}\right)$ and orbital moments $\left(m_{\mathrm{L}}\right)$ for $x=0,1$, and 2 for the experimentally found $\mathrm{AFM}$ structures within $\mathrm{GGA}+\mathrm{SOC}+U$.

\begin{tabular}{|c|c|c|c|c|c|}
\hline \multirow[b]{2}{*}{$x$} & \multicolumn{2}{|c|}{0} & \multirow[b]{2}{*}{1} & \multirow[b]{2}{*}{$2\left(P 2_{1} / n\right)$} & \multirow[b]{2}{*}{$2(I 2 / m)$} \\
\hline & FLL & AMF & & & \\
\hline$m_{\mathrm{S}}(\mathrm{Co})\left(\mu_{\mathrm{B}} /\right.$ atom $)$ & 2.68 & 1.84 & 2.67 & 3.05 & 2.99 \\
\hline$m_{\mathrm{L}}(\mathrm{Co})\left(\mu_{\mathrm{B}} /\right.$ atom $)$ & 0.17 & 0.17 & 0.16 & 0.11 & 0.10 \\
\hline$m_{\mathrm{S}}(\operatorname{Ir})\left(\mu_{\mathrm{B}} /\right.$ atom $)$ & 0.44 & -0.10 & 0.77 & 0.80 & 0.85 \\
\hline$m_{\mathrm{L}}(\operatorname{Ir})\left(\mu_{\mathrm{B}} /\right.$ atom $)$ & 0.43 & -0.01 & 0.29 & 0.16 & 0.17 \\
\hline
\end{tabular}

bital occupancy for $3 d$ Co in the vicinity of the desired AFM moment of $1.7 \mu_{\mathrm{B}} / \mathrm{Co}$ was forced with the FLL method. Apart from the HS state discussed above another LS state (also $\mathrm{Co}^{2+}$ ) with zero magnetic moment could be stabilized but a solution for the desired occupation did not converge. Therefore, another variant of the $\mathrm{GGA}+U$ approximation was used, namely, the "around mean-field" (AMF) method (Ref. 45), known to reduce the magnetic moment of the respective ion in certain cases. ${ }^{49}$ With this method a convergent solution could be found with an AFM moment of $1.84 \mu_{\mathrm{B}} / \mathrm{Co}$ (orbital moment: $0.17 \mu_{\mathrm{B}} / \mathrm{Co}$ ) with $U(\mathrm{Ir})$ $=2.72 \mathrm{eV}$ as above, but with a reduced $U$ for Co of $3.4 \mathrm{eV}$ (0.25 Ry). However, the magnetic moments of Ir are aligned antiparallel in contrast to the FLL results, where the alignment of the magnetic moments of $\mathrm{Co}$ and Ir becomes parallel, independent of the initial spin configuration (Table IX). Higher $U$ values with the AMF method resulted either in larger magnetic moments (for initial spin moments of Co larger than $2.6 \mu_{\mathrm{B}}$ ) or in smaller magnetic moments below $1 \mu_{\mathrm{B}} / \mathrm{Co}$ (for initial spin moments of Co smaller than 2.6 $\mu_{\mathrm{B}}$ ). In all cases the oxidation state of Co tends to be +2 . For $x$ $=1$ and 2 the calculated magnetic moments (HS) of Co with the FLL method agree well with the NPD results, taking the effect of finite temperature $(3 \mathrm{~K})$ and the site disorder ${ }^{50}$ on the $B / B^{\prime}$ sites into account. In case of $x=0$ the noncollinearity could also contribute to a further reduction in the magnetic moments. With an increasing degree of La substitution by $\mathrm{Sr}$ also a possible transition between the intermediate and high-spin state takes place for $\mathrm{Co}$, in addition to the obvious increase in the Co oxidation state. Note that spin and orbital contributions to the magnetic moments have always the same sign for both Co and Ir ions along the whole series. This is a remarkable difference to double perovskites with ferromagnetic ordering having high Curie temperatures (above room temperature) like the Re-based compounds $A_{2} B \mathrm{ReO}_{6}$ with $A=\mathrm{Ca}, \mathrm{Sr}$, or $\mathrm{Ba}$ and $B=\mathrm{Fe}$ or $\mathrm{Cr}$, where spin and orbital contributions on the $\mathrm{Re}$ site have always different signs. ${ }^{51}$

\section{CONCLUSIONS AND OUTLOOK}

The series $\mathrm{La}_{2-x} \mathrm{Sr}_{x} \mathrm{CoIrO}_{6}$ is a suitable model system for the effect of structural and chemical degrees of freedom on the resulting physical properties of double perovskites. The sequence of structural phase transitions dependent on composition and temperature was revealed. Three different types of magnetic structures are discussed with respect to electronic structure calculations. A reduction in the band gap is established with increasing $\mathrm{Sr}$ content, and a significant influence of $B / B^{\prime}$-site cation disorder on the electronic transport properties is proposed based on the results for $\mathrm{Sr}_{2}\left(\mathrm{Co}_{0.85} \mathrm{Ir}_{0.15}\right)\left(\mathrm{Ir}_{0.85} \mathrm{Co}_{0.15}\right) \mathrm{O}_{6}$. Competing interactions lead to $\mathrm{NCM}$, most pronounced in $\mathrm{La}_{2} \mathrm{CoIrO}_{6}$. The electronic structure calculations were performed for both AFM and FM configurations, which gave very similar total energies and thus support NCM by a sensitive preference of one of the possible states. Preliminary calculations for $\mathrm{La}_{2} \mathrm{CoIrO}_{6}$ were performed using the software code WIENNCM in order to include NCM. ${ }^{52}$ They showed $d$-band occupations and band gaps are similar to the reported ones for collinear configurations. The details of DFT calculations including NCM will be published separately. ${ }^{53}$ With increasing Sr content the ferromagnetic components are considerably reduced, so that the collinear approximations become more and more appropriate with increasing $x$. Further investigations will also focus on single-crystal magnetization studies and the effect of oxygen deficiency in $\mathrm{Sr}_{2} \mathrm{CoIrO}_{6-\delta}$.

\section{ACKNOWLEDGMENTS}

We are thankful to U. Nitzsche for technical assistance related to computer resources at IFW Dresden. This work has benefited from the use of the neutron powder diffractometer SPODI at FRM-II in Munich, funded by the German Bundesminister fuer Bildung und Forschung (BMBF) under Grant No. 03FU7DAR. The investigation of electronic and magnetic structures of complex Co oxides is supported by the Deutsche Forschungsgemeinschaft (Grant No. DFG FU125/50) within the Research Priority Programme SPP1178. 
*FAX: +49 3514659 452; n.narayanan@ifw-dresden.de

${ }^{1}$ K.-I. Kobayashi, T. Kimura, H. Sawada, K. Terakura, and Y. Tokura, Nature (London) 395, 357 (1998).

${ }^{2}$ H. Kato, T. Okuda, Y. Okimoto, Y. Tomioka, Y. Takenoya, A. Ohkubo, M. Kawasaki, and Y. Tokura, Appl. Phys. Lett. 81, 328 (2002).

${ }^{3}$ H. Kato, T. Okuda, Y. Okimoto, Y. Tomioka, K. Oikawa, T. Kamiyama, and Y. Tokura, Phys. Rev. B 65, 144404 (2002).

${ }^{4}$ F. Galasso and W. Darby, Inorg. Chem. 4, 71 (1965).

${ }^{5}$ G. Blasse, J. Inorg. Nucl. Chem. 27, 993 (1965).

${ }^{6}$ A. V. Powell, J. G. Gore, and P. D. Battle, J. Alloys Compd. 201, 73 (1993).

${ }^{7}$ M. Uhl, S. Matar, and B. Siberchicot, J. Magn. Magn. Mater. 187, 201 (1998).

${ }^{8}$ R. C. Currie, J. F. Vente, E. Frikkee, and D. J. W. Ijdo, J. Solid State Chem. 116, 199 (1995).

${ }^{9}$ G. Demazeau, B. Siberchicot, S. Matar, C. Gayet, and A. Largeteau, J. Appl. Phys. 75, 4617 (1994).

${ }^{10}$ M. Gateshki, J. M. Igartua, and E. Hernandez-Bocanegra, J. Phys.: Condens. Matter 15, 6199 (2003).

${ }^{11}$ Q. Zhou, B. J. Kennedy, and M. M. Elcombe, J. Solid State Chem. 180, 541 (2007).

${ }^{12}$ M. Knapp, C. Baehtz, H. Ehrenberg, and H. Fuess, J. Synchrotron Radiat. 11, 328 (2004).

${ }^{13}$ M. Knapp, V. Joco, C. Baehtz, H. H. Brecht, A. Berghaeuser, H. Ehrenberg, H. von Seggern, and H. Fuess, Nucl. Instrum. Methods Phys. Res. A 521, 565 (2004).

${ }^{14}$ J. Ihringer and A. Küster, J. Appl. Crystallogr. 26, 135 (1993).

${ }^{15}$ J. Rodríguez-Carvajal, Physica B 192, 55 (1993).

${ }^{16}$ P. M. Woodward, Acta Crystallogr., Sect. B: Struct. Sci. 53, 32 (1997).

${ }^{17}$ P. Blaha, K. H. Schwarz, G. Madsen, D. Kvasnicka, and J. Luitz, WIEN2k, An Augmented Plane Wave plus Local Orbitals Program for Calculating Crystal Properties (TU Wien, Austria, 2001).

${ }^{18}$ J. P. Perdew, K. Burke, and M. Ernzerhof, Phys. Rev. Lett. 77, 3865 (1996).

${ }^{19}$ J. Kuneš, P. Novák, M. Diviš, and P. M. Oppeneer, Phys. Rev. B 63, 205111 (2001).

${ }^{20}$ R. F. W. Bader, Atoms in Molecules-A Quantum Theory (Clarendon Press, Oxford, UK, 1990).

${ }^{21}$ R. Vidya, P. Ravindran, K. Knizek, A. Kjekshus, and H. Fjellvag, Inorg. Chem. 47, 6608 (2008).

${ }^{22}$ C. J. Howard, B. J. Kennedy, and P. M. Woodward, Acta Crystallogr., Sect. B: Struct. Sci. 59, 463 (2003).

${ }^{23}$ G. Popov, M. Greenblatt, and M. Croft, Phys. Rev. B 67, 024406 (2003).

${ }^{24}$ D. Serrate, J. M. De Teresa, and M. R. Ibarra, J. Phys.: Condens. Matter 19, 023201 (2007).

${ }^{25}$ W. T. Pennington, J. Appl. Crystallogr. 32, 1028 (1999).

${ }^{26}$ I. D. Brown and D. Altermatt, Acta Crystallogr., Sect. B: Struct. Sci. 41, 244 (1985).

${ }^{27}$ I. D. Brown, accumulated table of bond valence parameters, bvparm2006.cif, 2006 (unpublished).

${ }^{28}$ R. D. Shannon, Acta Crystallogr., Sect. A: Cryst. Phys., Diffr.,
Theor. Gen. Crystallogr. 32, 751 (1976).

${ }^{29}$ L. Ortega-San Martin, J. P. Chapman, E. Hernández-Bocanegra, M. Insausti, M. I. Arriortua, and T. Rojo, J. Phys.: Condens Matter 16, 3879 (2004).

${ }^{30}$ D. Serrate, J. M. De Teresa, P. A. Algarabel, J. Galibert, C. Ritter, J. Blasco, and M. R. Ibarra, Phys. Rev. B 75, 165109 (2007).

${ }^{31}$ M. Sikora, O. Mathon, P. van der Linden, J. M. Michalik, J. M. de Teresa, C. Kapusta, and S. Pascarelli, Phys. Rev. B 79, 220402(R) (2009).

${ }^{32}$ V. Yu. Irkhin, J. Magn. Magn. Mater. 258-259, 228 (2003).

${ }^{33}$ M. Retuerto, M. J. Martínez-Lope, M. García-Hernández, M. T. Fernández-Díaz, and J. A. Alonso, Eur. J. Inorg. Chem. 2008, 588 (2007).

${ }^{34}$ Z. H. Han, H. E. Mohottala, J. I. Budnick, W. A. Hines, P. W. Klamut, B. Dabrowski, and M. Maxwell, J. Phys.: Condens. Matter 18, 2273 (2006).

${ }^{35}$ K. Oikawa, T. Kamiyama, H. Kato, and Y. Tokura, J. Phys. Soc. Jpn. 72, 1411 (2003).

${ }^{36}$ P. D. Battle, J. B. Goodenough, and R. Price, J. Solid State Chem. 46, 234 (1983).

${ }^{37}$ J. B. Goodenough, Magnetism and the Chemical Bond (Wiley, New York, London, 1963), p. 177, Table XII.

${ }^{38}$ Jan-Willem G. Bos and J. P. Attfield, Phys. Rev. B 69, 094434 (2004).

${ }^{39}$ Jan-Willem G. Bos and J. P. Attfield, Phys. Rev. B 70, 174434 (2004).

${ }^{40}$ N. F. Mott, Philos. Mag. 19, 835 (1969).

${ }^{41}$ C. Michel, S. D. Baranovskii, P. J. Klar, and P. Thomas, Appl. Phys. Lett. 89, 112116 (2006).

${ }^{42}$ J.-W. G. Bos and J. P. Attfield, Chem. Mater. 16, 1822 (2004).

${ }^{43}$ P. Tomeš, J. Hejtmánek, and K. Knížek, Solid State Sci. 10, 486 (2008).

${ }^{44}$ I. V. Solovyev, P. H. Dederichs, and V. I. Anisimov, Phys. Rev. B 50, 16861 (1994).

${ }^{45}$ M. T. Czyżyk and G. A. Sawatzky, Phys. Rev. B 49, 14211 (1994)

${ }^{46}$ V. I. Anisimov, I. V. Solovyev, M. A. Korotin, M. T. Czyżyk, and G. A. Sawatzky, Phys. Rev. B 48, 16929 (1993).

${ }^{47}$ D. Niebieskikwiat, R. D. Sanchez, A. Caneiro, L. Morales, M. Vasquez-Mansilla, F. Rivadulla, and L. E. Hueso, Phys. Rev. B 62, 3340 (2000).

${ }^{48}$ H. Wu and T. Burnus, Phys. Rev. B 80, 081105(R) (2009).

${ }^{49}$ E. R. Ylvisaker, W. E. Pickett, and K. Koepernik, Phys. Rev. B 79, 035103 (2009).

${ }^{50}$ L. Balcells, J. Navarro, M. Bibes, A. Roig, B. Martınez, and J. Fontcuberta, Appl. Phys. Lett. 78, 781 (2001).

${ }^{51}$ A. Winkler, N. Narayanan, D. Mikhailova, K. G. Bramnik, H. Ehrenberg, H. Fuess, G. Vaitheeswaran, V. Kanchana, F. Wilhelm, A. Rogalev, A. Kolchinskaya, and L. Alff, New J. Phys. 11, 073047 (2009).

${ }^{52}$ R. Laskowski, G. K. H. Madsen, P. Blaha, and K. H. Schwarz, Phys. Rev. B 69, 140408(R) (2004).

${ }^{53}$ N. Narayanan, R. Laskowski, D. Mikhailova, H. Ehrenberg, A. Senyshyn, P. Blaha, K. H. Schwarz, and H. Fuess (unpublished) 